\title{
Differential expression of genes identified from Poncirus trifoliata tissue inoculated with CTV through EST analysis and in silico hybridization
}

\author{
Mariângela Cristofani-Yaly ${ }^{1}$, Irving J. Berger ${ }^{1}$, Maria Luisa P.N. Targon ${ }^{1}$, Marco A. Takita ${ }^{1}$, \\ Sílvia de O. Dorta ${ }^{1}$, Juliana Freitas-Astúa ${ }^{1,2}$, Alessandra A. de Souza ${ }^{1}$, \\ Raquel L. Boscariol-Camargo ${ }^{1}$, Marcelo S. Reis ${ }^{1}$ and Marcos A. Machado ${ }^{1}$ \\ ${ }^{1}$ Laboratório de Biotecnologia, Centro APTA Citros Sylvio Moreira, Instituto Agronômico de Campinas, \\ Cordeirópolis, SP, Brazil. \\ ${ }^{2}$ Embrapa Milho e Sorgo, Sete Lagoas, MG, Brazil.
}

\begin{abstract}
Citrus is the most important fruit crop in Brazil and Citrus tristeza virus (CTV) is considered one of the most important pathogens of citrus. Most citrus species and varieties are susceptible to CTV infection. However, Poncirus trifoliata, a close relative of citrus, is resistant to the virus. In order to better understand the responses of citrus plants to the infection of CTV, we constructed expressed sequence tag (EST) libraries with tissues collected from Poncirus trifoliata plants, inoculated or not with Citrus tristeza virus at 90 days after inoculation, grafted on Rangpur lime rootstocks. We generated 17,867 sequence tags from Poncirus trifoliata inoculated (8,926 reads) and not (8,941 reads) with a severe CTV isolate. A total of 2,782 TCs (Tentative Consensi sequences) were obtained using both cDNA libraries in a single clusterization procedure. By the in silico hybridization approach, 289 TCs were identified as differentially expressed in the two libraries. A total of 121 TCs were found to be overexpressed in plants infected with CTV and were grouped in 12 primary functional categories. The majority of them were associated with metabolism and defense response. Some others were related to lignin, ethylene biosynthesis and PR proteins. In general, the differentially expressed transcripts seem to be somehow involved in secondary plant response to CTV infection.
\end{abstract}

Key words: citrus, disease resistance, Citrus tristeza virus, biotic stress.

Received: November 7, 2006; Accepted: May 14, 2007.

\section{Introduction}

Citrus is the most important fruit crop in Brazil. Among all its pathogens, Citrus tristeza virus (CTV) is considered one of the most important ones. This virus is an aphid-transmitted, positive sense, single-stranded RNA member of the Closteroviridae. Most citrus species and varieties are susceptible to $\mathrm{CTV}$ infection. However, Poncirus trifoliata, a close relative of citrus, is resistant to CTV. There are other citrus relatives like Severinia buxifolia (Poir) Ten and Swinglea glutinosa (Blanco) Merr that are also resistant to infection by most CTV strains (Albiach-Marti et al., 2004).

CTV resistance seems to be a single gene dominant trait (Ctv, Gmitter et al., 1996), but according to AlbiachMarti et al. (2004) this resistance is modified by a second gene (Ctm) (Mestre et al., 1997). Moreover, plants which are heterozygous for $C t v$ are resistant to most CTV isolates,

Send correspondence to Mariângela Cristofani-Yaly. Centro APTA Citros Sylvio Moreira, Instituto Agronômico de Campinas, Caixa Postal 4, 13490-970 Cordeirópolis, SP, Brazil. E-mail: mariangela@centrodecitricultura.br. but may allow local movement in the absence of $\mathrm{Ctm}$ (Mestre et al., 1997). It has been suggested that Ctv resistance is constitutive, preventing some early step in the infection process (Albiach-Marti et al., 2004).

To complete its cycle, the virus undergoes a multistep process that includes entry into plant cells, uncoating of nucleic acid, translation of viral proteins, replication of viral nucleic acid, assembly of progeny virion, cell-to-cell movement, systemic movement and plant-to-plant transmission (Kang et al., 2005).

Albiach-Marti et al. (2004) showed that a range of biologically and genetically distinct CTV isolates were able to replicate and form infectious viral particles in protoplasts obtained from P. trifoliata and Citrus x Poncirus hybrid plants (which contained the Ctv resistance gene) and in protoplasts from $S$. buxifolia and $S$. glutinosa plants. According to the authors, this suggests that the Poncirus resistance affects a viral step subsequent to replication and assembly of viral particles. Nevertheless, it should be noted that these data do not eliminate the possibility that resistance is due to a hypersensitive response (HR) since it could 
happen without visible necrosis and also, replication in protoplasts is possible in incompatible interactions (Albiach-Marti et al., 2004).

In order to better understand the responses of $P$. trifoliata plants to the infection of CTV, we constructed expressed sequence tag (EST) libraries from Poncirus trifoliata plants inoculated or not with a severe Citrus tristeza virus (CTV) grafted on Rangpur lime rootstocks. The reads were analyzed using bioinformatics tools to generate a picture of the defense response of Poncirus trifoliata to CTV infection.

\section{Material and Methods}

\section{Plant material and construction of libraries}

Buds of Pêra sweet orange (Citrus sinensis Osbeck) infected with a severe isolate of CTV and free of virus were grafted on Poncirus trifoliata cv. Rubidoux previously grafted on Rangpur lime (Citrus limonia Osbeck) rootstocks. The infected and non-infected buds of sweet orange were left as a continuous source of inoculum and control, respectively. Approximately $1-3 \mathrm{~g}$ of the leaf tissue from Poncirus trifoliata were collected from each treatment, 90 days after inoculation. Plants were kept under greenhouse conditions.

The libraries were prepared from mRNA isolated from leaves collected from both non-inoculated and CTV inoculated specimens at the same developmental stage. RNA extraction, cDNA and sequencing were done according to Targon et al. (in this issue).

\section{EST sequencing and data analysis}

Sequencing was carried out using the Big Dye Terminator v.3 Kit as described by the manufacturer (Perkin-Elmer). Products were separated by capillary electrophoresis using an ABI 3700 sequencer (Applied Biosystems).

\section{In silico hibridization and functional annotation}

For comparison of the libraries, we performed an in silico hybridization analysis. The in silico hybridization methodology included a clusterization of all transcripts from both libraries, using the CAP3 tool (Huang and Madan, 1999), with the default parameters. Furthermore, for each tentative consensus (TC) sequence, the relative abundance of transcripts was calculated, using a correction factor of 10,000 for normalization. The differential in silico expression was then evaluated using statistic verification (Audic and Claverie, 1997). We considered differential expression as the possibility of a random transcript abundance distribution, for a given TC, to be equal to or lower than 5\% $(\mathrm{P}$-value $\leq 0.05)$. Automatic categorization over the tentative consensi (TC) was performed as well, using the $\mathrm{Mu}-$ nich Center for Proteins and Sequences Functional Categories (MIPS) v. 1.3 (http://mips.gsf.de). Comparative genomics was carried out through TC comparison against the GenBank protein database, using the Blastall implementation of the BLAST algorithm (Altschul et al., 1997). More details on the bioinformatics analyses can be found at Reis et al. (in this issue).

\section{Results and Discussion}

\section{Differential expression and functional annotation}

We generated a total of 17,867 ESTs from Poncirus trifoliata, with 8,926 reads coming from CTV inoculated and 8,941 reads from the control source. A total of 2,782 TCs (Tentative Consensi sequences) were obtained using both cDNA libraries in a single clusterization procedure. Through the in silico hybridization approach, 289 TCs were identified as differentially expressed in the two libraries. A total of 121 TCs out of these were found to be putatively overexpressed in plants infected with CTV (Table 1), while 168 TCs were potentially underexpressed. The 121 overexpressed TCs were grouped in 12 primary functional categories and the 168 underexpressed TCs were grouped in 16 categories (data not shown). An overview of the functional categorization of the putative overexpressed TCs with known or predicted functions is presented in Figure 1.

The largest set of genes $(29.75 \%)$ was assigned to the metabolism category, while genes involved in transcription constituted the smallest group, comprising less than $2.47 \%$ of the genes. Genes involved in signal transduction and protein destination/storage were $4.95 \%$. Genes implicated in stress/defense response constituted $15.70 \%$ of the infected cDNA collection. Proteins with unknown functions corresponded to $21.48 \%$. They were similar to already sequenced plant genes of unknown function and might be an additional source of genes participating in the expression of citrus in response to biotic stresses.

\section{General responses to the Citrus tristeza virus}

\section{Synthesis of phenylpropanoids}

We identified several genes involved in the biosynthesis of defense-related secondary metabolites (phenylpropanoids and phytoalexins). Together, these metabolites function in a variety of defense-related processes, including the induction of wound response, antimicrobial and antifungal defense, and antioxidant defense (Verica et al., 2004).

In the present work, we identified six putative proteins encoded by the overexpressed genes related to phenylpropanoids: 4-coumarate-CoA ligase, anthralinate N-benzoyltransferase, flavonol synthase, cinnamoyl CoAredutase, caffeic acid-O-methyltransferase and anthocyanin 5-aromatic acyltransferase. The 4-coumarate-CoA ligases belong to a group of enzymes necessary for maintaining a continuous metabolic flux for the biosynthesis of plant phenylpropanoids, such as lignin and flavonoids, which are essential for the survival of plants. Thus, hydro- 
Table 1 - Functional categories of genes detected as overexpressed in Poncirus trifoliata infected with Citrus tristeza virus at 90 days after inoculation.

\begin{tabular}{|c|c|c|c|c|c|}
\hline Functional category & Best blast match & Organism & Accession number & $\%$ identity & e-value \\
\hline \multicolumn{6}{|l|}{ 01. Metabolism } \\
\hline & Alanine-glyoxylate aminotransferase & Arabidopsis thaliana & AT2G13360 & 86 & 0.0 \\
\hline & Enolase & Ricinus communis & CAA82232.1 & 92 & e-179 \\
\hline & S-adenosylmethionine (adoMetDC2) & Arabidopsis thaliana & AT5G15950 & 67 & e-125 \\
\hline & Neutral invertase & Arabidopsis thaliana & AT1G56560 & 74 & 0.0 \\
\hline & 4-Coumarate -CoA ligase & Arabidopsis thaliana & AT1G65060 & 63 & $3 e-78$ \\
\hline & Phosphoribulokinase & Arabidopsis thaliana & AAN15338 & 92 & e-108 \\
\hline & Proline iminopeptidase & Arabidopsis thaliana & AT2G14260 & 83 & $1 \mathrm{e}-71$ \\
\hline & Phosphoribosylglycinamide Formyltransferase 2 & Bordetella parapertussis & NP886172.1 & 85 & $3 e-13$ \\
\hline & Thiazole biosynthetic enzyme precursor & Citrus sinensis & cab05370.1 & 95 & e-118 \\
\hline & Thiazole biosynthetic enzyme precursor & Citrus sinensis & cab05370.1 & 84 & e-156 \\
\hline & ACC Synthase & Arabidopsis thaliana & AT5G51690 & 67 & e-129 \\
\hline & ACC Oxidase & Arabidopsis thaliana & AT1G05010 & 75 & e-148 \\
\hline & Ethylene forming enzyme (ACO) & Arabidopsis thaliana & AAM613662.1 & 67 & e- 85 \\
\hline & Anthranilate N-Benzoyltransferase & Arabidopsis thaliana & AT5G01210 & 65 & $2 \mathrm{e}-82$ \\
\hline & Flavonol synthase & Oryza sativa & XP-482984.1 & 45 & $2 \mathrm{e}-68$ \\
\hline & Cinnamoyl CoA reductase & Arabidopsis thaliana & AT2G02400 & 67 & e-129 \\
\hline & Caffeic acid O-Methyltransferase & Arabidopsis thaliana & AT3G53140 & 74 & e-155 \\
\hline & Xyloglucan endo-transglycosylase & Arabidopsis thaliana & AT1G14720 & 69 & e-135 \\
\hline & Fructose-biphosphate aldolase & Arabidopsis thaliana & AT4G26530 & 75 & $1 \mathrm{e}-22$ \\
\hline & Anthocyanin5-aromatic acyltransferase & Arabidopsis thaliana & AT3G29590 & 39 & $3 e-72$ \\
\hline & UMP synthase & Arabidopsis thaliana & AT3G54470 & 85 & e-111 \\
\hline & Putative glyoxysomal malate dehydrogenase & Arabidopsis thaliana & AT2G36790 & 50 & $4 e-61$ \\
\hline & Cobalamin Synthase & Arabidopsis thaliana & NP-173974 & 77 & $6 e-82$ \\
\hline & $\begin{array}{l}\text { Methylthioadenosine/S-adenosyl homocysteine } \\
\text { nucleosidase }\end{array}$ & Oryza sativa & NP-910292.1 & 71 & $4 e-84$ \\
\hline & Phosphate/phosphoenolpyruvate & Arabidopsis thaliana & AT5G33320 & 47 & $2 \mathrm{e}-35$ \\
\hline & Phosphoenolpyruvate carboxylase & Glycine $\max$ & AAS67005.1 & 86 & 0.0 \\
\hline & Putative Prolyl endopeptidase & Arabidopsis thaliana & AAL86330.1 & 72 & 0.0 \\
\hline & Sucrose synthase & Citrus unshiu & BAA 88904.1 & 98 & e-117 \\
\hline & Sedoheptulose-Biphosphatase & Arabidopsis thaliana & AT3G55800 & 82 & 0.0 \\
\hline & 3-ketoacyl-CoA thiolase & Arabidopsis thaliana & AT2G33150 & 89 & e-174 \\
\hline & Inositol 1,3,4-Triphosphate & Arabidopsis thaliana & AT4G08170 & 90 & $1 e-75$ \\
\hline & Flavonol synthase & Oryza sativa & XP-482984.1 & 45 & $2 \mathrm{e}-68$ \\
\hline & Sucrose synthase & Citrus unshiu & BAA88904.1 & 98 & e-117 \\
\hline & UDP-Glucoyl transferase & Arabidopsis thaliana & AT2G36790 & 50 & $4 e-61$ \\
\hline & UDP-Glucose Glucosyltransferase & Rhodiola sachalinensis & AAS55083.1 & 56 & e-110 \\
\hline & Glycosyltransferase NTGT5a & Nicotiana tabacum & BAD93689.1 & 65 & $1 e-87$ \\
\hline \multicolumn{6}{|l|}{ 02. Energy } \\
\hline & Chlorophyll A/B-binding protein & Arabidopsis thaliana & AT4G10340 & 83 & $5 e-96$ \\
\hline & Photosystem II Polypeptide & Arabidopsis thaliana & AAM20194.1 & 82 & $2 \mathrm{e}-57$ \\
\hline & Ribulose 1,5-Biphosphate Carboxylase & Manihot esculenta & AAF06101.1 & 81 & $1 e-42$ \\
\hline & $\begin{array}{l}\text { NADP-Dependent Glyceraldehyde-3-phosphate } \\
\text { Dehydrogenase }\end{array}$ & Arabidopsis thaliana & AT2G24270 & 90 & 0.0 \\
\hline & Protein I Photosystem II oxygen-evolving & Arabidopsis thaliana & AT3G50820 & 79 & e-155 \\
\hline \multicolumn{6}{|l|}{ 04. Transcription } \\
\hline & Ein3-like & Cucumis melo & BAB64345.1 & 55 & $5 e-78$ \\
\hline & RNA polymerase Sigma 70 & Arabidopsis thaliana & AT2G36990 & 58 & $1 e-44$ \\
\hline & Homeobox-leucine zipper protein HAT5 & Arabidopsis thaliana & AT3G01470 & 39 & $1 e-44$ \\
\hline
\end{tabular}


Table 1 (cont.)

\begin{tabular}{|c|c|c|c|c|c|}
\hline Functional category & Best blast match & Organism & Accession number & $\%$ identity & e-value \\
\hline \multicolumn{6}{|l|}{ 05. Protein synthesis } \\
\hline & Translation initiation factor eIF-2 Beta chain & Arabidopsis thaliana & AT5G20920 & 76 & e-113 \\
\hline & 30 Ribosomal protein S5 & Arabidopsis thaliana & AT2G33800 & 69 & e-109 \\
\hline & 60S Ribosomal protein L1 & Arabidopsis thaliana & AT3G09630 & 87 & e-104 \\
\hline & 50S Ribosomal protein L13 & Arabidopsis thaliana & AAD30573.1 & 66 & $3 e-90$ \\
\hline \multicolumn{6}{|c|}{ 06. Protein fate (folding, modification, destination) } \\
\hline & Hydroxypyruvate reductase (HPR) & Arabidopsis thaliana & AT1G68010 & 90 & 0.0 \\
\hline & FtsH chloroplast protease & Arabidopsis thaliana & AT2G30950 & 86 & 0.0 \\
\hline & Expressed protein & Arabidopsis thaliana & NP 178048.1 & 33 & $3 e-21$ \\
\hline & Ketoacyl-CoA & Arabidopsis thaliana & AT2G33150 & 89 & e-174 \\
\hline & ATP-dependent Clp protease & Arabidopsis thaliana & AT5G50920 & 92 & 0.0 \\
\hline & Transformer-SR ribonucleoprotein putative & Arabidopsis thaliana & AT1G07350 & 51 & $1 \mathrm{e}-40$ \\
\hline \multicolumn{6}{|c|}{ 08. Cellular transport and transport mechanism } \\
\hline & $\mathrm{ABC}$ transporter & Arabidopsis thaliana & NP-188762.2 & 78 & 0.0 \\
\hline & $\mathrm{ABC}$ Transporter protein 1-Like & Arabidopsis thaliana & AT5G64840 & 86 & e-132 \\
\hline & Peroximal membrane related protein & Arabidopsis thaliana & NP564615.1 & 87 & $7 e-84$ \\
\hline & Rieske iron-sulfur protein & Nicotiana tabacum & AAA20832.1 & 73 & $1 e-94$ \\
\hline & Sulfate transporter 2 & Lycopersicon esculentum & AAK27688.1 & 80 & 0.0 \\
\hline & ADP-rybosylation factor-like protein & Arabidopsis thaliana & AT3G62290 & 97 & e-100 \\
\hline \multicolumn{6}{|c|}{ 10. Cellular communication/Signal transduction mechanism } \\
\hline & BIS (5-Adenosyl triphosphatase; histidine triad) & Arabidopsis thaliana & AT5G58240 & 67 & $3 e-55$ \\
\hline & Rab-type small GTP-binding & Arabidopsis thaliana & AT5G45750 & 100 & $7 e-12$ \\
\hline & Ras-related GTP-binding & Arabidopsis thaliana & AT5G45130 & 56 & $8 e-99$ \\
\hline & CONSTAINS- like- B Box Zinc Finger & Arabidopsis thaliana & AT5G57660 & 54 & e-103 \\
\hline & Zinc Finger & Arabidopsis thaliana & NP-197938.2 & 72 & e-133 \\
\hline & 14-3-3 Protein GF14 & Arabidopsis thaliana & AT5G65430 & 86 & e-117 \\
\hline \multicolumn{6}{|c|}{ 11. Cell rescue, defense and virulence } \\
\hline & NADPH Oxydase & Arabidopsis thaliana & AT5G49730 & 59 & $3 e-59$ \\
\hline & Germin-like protein & Arabidopsis thaliana & AT1G72610 & 68 & $6 e-76$ \\
\hline & Papain-like Cysteine proteinase & Gossypium hirsuntum & CAE54306.1 & 75 & e-113 \\
\hline & Chitinase & Citrus sinensis & CAA938471 & 89 & e-107 \\
\hline & Ankyrin & Vitis aestivalis & AAQ96339.1 & 66 & $4 e-85$ \\
\hline & NADPH-Ferrihemoprotein reductase (ATR2) & Arabidopsis thaliana & AT4G30210 & 81 & 0.0 \\
\hline & N-Rich protein & Glycine $\max$ & CAI44933.1 & 60 & e-110 \\
\hline & SRG1 & Arabidopsis thaliana & AT1G17020 & 56 & e-116 \\
\hline & Miraculin-like protein 2 & Citrus paradisi & AAG38518.1 & 44 & $7 e-42$ \\
\hline & Miraculin-like protein 3 & Citrus paradisi & AAG38519.1 & 39 & $4 e-31$ \\
\hline & DNAJ & Arabidopsis thaliana & AT3G44110 & 84 & 0.0 \\
\hline & High molecular weight heat shock protein & Malus $x$ domestica & AAF34134 & 93 & e-147 \\
\hline & TCP1-chaperonin cofactor A & Arabidopsis thaliana & AAM63030.1 & 82 & $6 e-46$ \\
\hline & Cytochrome P450 & Arabidopsis thaliana & AT3G52970 & 40 & $4 e-50$ \\
\hline & Peroxidase prxr1 & Arabidopsis thaliana & AT4G21960 & 83 & e-159 \\
\hline & Type I proteinase inhibitor-like protein & Citrus paradisi & AAN76363.1 & 97 & $4 e-65$ \\
\hline & Resistance protein & Arabidopsis thaliana & AT5G52780 & 45 & $3 e-29$ \\
\hline & Putative auxin-induced protein & Arabidopsis thaliana & AT1G23740 & 73 & e-125 \\
\hline & LLS1-like protein & Arabidopsis thaliana & AAR05798.1 & 61 & e-140 \\
\hline
\end{tabular}


Table 1 (cont.)

\begin{tabular}{|c|c|c|c|c|c|}
\hline Functional category & Best blast match & Organism & Accession number & $\%$ identity & e-value \\
\hline \multicolumn{6}{|c|}{ 40. Subcellular localization } \\
\hline & $\mathrm{CP} 12$ protein & Arabidopsis thaliana & AT3G62410 & 56 & $3 e-31$ \\
\hline & Coatomer complex subunit & Arabidopsis thaliana & AT1G52360 & 78 & e-156 \\
\hline \multicolumn{6}{|c|}{ 63. Protein with binding function or cofactor requirement } \\
\hline & NADP/NADP binding & Arabidopsis thaliana & AT1G42970 & 85 & 0.0 \\
\hline & DNA binding protein & Arabidopsis thaliana & AAN13013.1 & 60 & e-156 \\
\hline & Arabidopsis dynamin like protein ADL2 & Arabidopsis thaliana & AT4G33650 & 59 & $3 e-34$ \\
\hline & RNA binding protein & Arabidopsis thaliana & AT1G09340 & 78 & e-143 \\
\hline \multicolumn{6}{|c|}{ 67. Transport facilitation } \\
\hline & Aquaporin & Arabidopsis thaliana & AT3G01280 & 73 & e-115 \\
\hline & Aquaporin & Arabidopsis thaliana & AT2G45960 & 89 & $\mathrm{e}-150$ \\
\hline & Aquaporin & Arabidopsis thaliana & AT2G36830 & 80 & e-115 \\
\hline & Aldo/Keto reductase & Fragaria $x$ ananassa & AAV28174.1 & 61 & e-115 \\
\hline \multicolumn{6}{|c|}{ 99. Unclassified protein } \\
\hline & & Arabidopsis thaliana & AAM63493.1 & 59 & $1 \mathrm{e}-39$ \\
\hline & & Arabidopsis thaliana & AT3G09050 & 63 & $1 \mathrm{e}-86$ \\
\hline & & Arabidopsis thaliana & AT1G44920 & 69 & $5 \mathrm{e}-80$ \\
\hline & & Arabidopsis thaliana & AT2G46820 & 64 & $2 e-57$ \\
\hline & & Arabidopsis thaliana & AT2G39570 & 59 & $1 \mathrm{e}-11$ \\
\hline & & Arabidopsis thaliana & AT3G58900 & 52 & $3 e-07$ \\
\hline & & Arabidopsis thaliana & AT2G16350 & 38 & $7 e-22$ \\
\hline & & Arabidopsis thaliana & AT4G11570 & 77 & $3 e-16$ \\
\hline & & Arabidopsis thaliana & AT3G52740 & 52 & $2 \mathrm{e}-25$ \\
\hline & & Arabidopsis thaliana & AT1G48090 & 74 & e-119 \\
\hline & & Arabidopsis thaliana & AT1G63610 & 72 & e-129 \\
\hline & & Arabidopsis thaliana & AT3G57890 & 71 & 0.0 \\
\hline & & Arabidopsis thaliana & AT2G03440 & 50 & $4 e-26$ \\
\hline & & Arabidopsis thaliana & AT1G74640 & 81 & e-130 \\
\hline & & Arabidopsis thaliana & AT4G32020 & 41 & $7 e-25$ \\
\hline & & Arabidopsis thaliana & AT2G44310 & 80 & $5 e-61$ \\
\hline & & Arabidopsis thaliana & AT3G07760 & 91 & $6 e-64$ \\
\hline & & Arabidopsis thaliana & AT1G15340 & 47 & $9 e-54$ \\
\hline & & Arabidopsis thaliana & AT2G35330 & 69 & $1 \mathrm{e}-64$ \\
\hline & & Arabidopsis thaliana & AT3G56360 & 42 & $1 \mathrm{e}-40$ \\
\hline & & Arabidopsis thaliana & AT3G22850 & 75 & e-110 \\
\hline & & Arabidopsis thaliana & AT1G09930 & 58 & e-112 \\
\hline & & Arabidopsis thaliana & AT5G53450 & 66 & 0.0 \\
\hline & & Arabidopsis thaliana & AT3G21360 & 74 & e-146 \\
\hline & & Arabidopsis thaliana & AT5G23950 & 43 & $1 \mathrm{e}-49$ \\
\hline & & Arabidopsis thaliana & AT3G06190 & 74 & e-108 \\
\hline
\end{tabular}

xycinnamoyl-CoA:shikimate /quinate hydroxycinnamoyl transferase seems to control the biosynthesis and turnover of major plant phenolic compounds such as lignin and chlorogenic acid. Benzoyl-CoA:anthranilate N-benzoyltransferase catalyzes the first committed reaction of phytoalexin biosynthesis in carnation (Dianthus caryophyllus L.) (Reinhard and Matern, 1989).
Only a few studies have demonstrated the antiviral activity of phenylpropanoids against plant viruses (Chong et al., 2002). However, a range of flavonoids inhibit the infectivity of Tobacco mosaic virus (TMV) (French et al., 1991). Up-regulation of the flavonol synthase encoding gene reported here may be involved in such a resistance mechanism in P. trifoliata against CTV. 


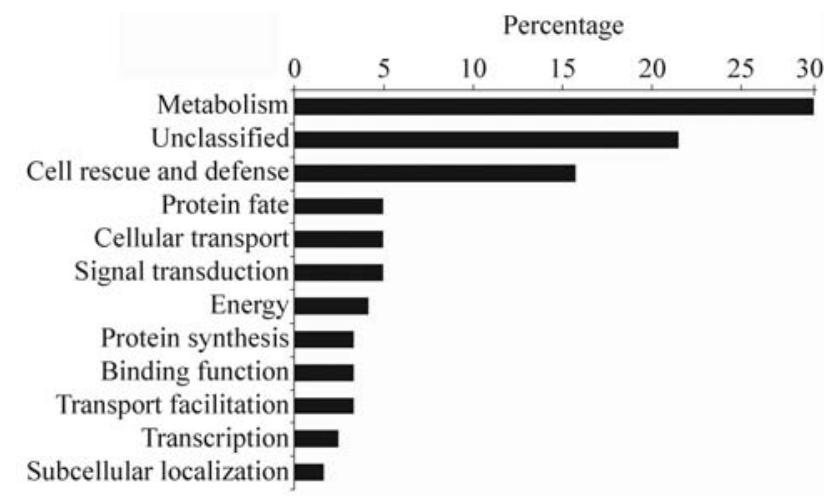

Figure 1 - Functional classification following MIPS categories (Munich Information Center for Protein Sequences) of expressed sequence tags (ESTs), identified as overexpressed in the library of CTV inoculated plants.

\section{Cell wall changes}

Cell wall reinforcement and thickening are associated with plant defense during resistance responses. In this study, we found two TCs (cinnamoyl-CoA reductase and caffeic acid-O-methyltransferase) whose expression is associated with lignification in several dicot species (Ye, 1997; Ye et al., 2001). Lignin is a complex phenolic polymer that reinforces the walls of certain cells in higher plants. Such reinforcement is an effective defense response against infection by pathogens (Kawasaki et al., 2006). In addition, many antimicrobial substances such as phytoalexins are known to be produced by the monolignol synthetic pathways. It is therefore likely that lignin and lignin-related compounds with antimicrobial activities cooperatively play important roles in disease resistance of various plant species. Lignin synthesis was induced in soybean leaves inoculated with Soybean mosaic virus (SMV) (Hajimorad and Hill, 2001).

According to Jaeck et al. (1992) the regulation of an enzyme involved in lignin biosynthesis, an O-methyltransferase occurred during the hypersensitive reaction of tobacco in interactions with TMV.

\section{Phytohormones}

In the present work we also identified four TCs (S-adenosylmethionine adoMetDC2) (SAM), ACC synthase, ACC oxidase and ethylene forming enzyme (ACO) whose expression is putatively overexpressed under conditions inducing ethylene biosynthesis (Gomez-Gomez and Carrasco, 1998). SAM serves as a precursor of the plant hormone ethylene, implicated in the control of numerous developmental processes (Kende, 1993). In a cDNA library, prepared from leaves of TMV-infected tobacco after TMV infection and subsequent recognition of the pathogen by the host, ethylene is produced by the conversion of S-adenosyl-L-methionine (SAM) into ACC. ACC is then converted into ethylene, carbon dioxide and cyanide. Ethylene production generates a molecular and genetic cascade of responses that lead to the induction of host defense- related genes (Knoester et al., 1995). In the present work, the up-regulation of ACC enzymes suggests a possible TMV-like interaction and, thus, indicates that ethylene participates in the response to CTV infection.

Additionally, a TC coding for a putative EIN3-like protein (an important component in ethylene signal transduction pathway) was identified, corroborating the possible participation of ethylene in the plant response to CTV infection.

\section{Defense-related genes}

The third largest functional category (accounting for $15.70 \%$ of the differentially expressed genes) was cell rescue, defense, cell death and ageing. This group includes putative homologs of ankyrin, NADPH oxydase, germin, papain-like cysteine proteinase, chitinase, NADPH-ferrihemoprotein reductase (ATR2), N-rich protein, SRG1, miraculin-like protein 2, miraculin-like protein 3, DNAJ, TCP1-chaperonin cofactor A, cytochrome P450, peroxidase prxr1 PR9, type I proteinase inhibitor-like protein, resistance protein, putative auxin-induced protein and LLS1-like protein.

A N-rich protein was found overexpressed in CTV inoculated plants in the present work. According to Ludwig and Tenhaken (2001), the NRP gene appears to be a new marker in early responses in plant disease resistance. The protein is located in the cell wall, with a very high content of asparagines and was, therefore, termed N-rich protein (NRP). The NRP-gene is not directly induced by salicylic acid or hydrogen peroxide, indicating a distinct and specific signal transduction pathway which is only activated during programmed cell death.

One of the putative TCs related to cell defense showed similarity to LLS1 (Lethal leaf spot-1) that has a role in cell death-suppression. LLS1 may act to prevent reactive oxidative species formation or serve to remove a cell death mediator to maintain chloroplast integrity and cell survival. Yang et al. (2004) demonstrated that the LLS1 protein is present constitutively in all photosynthetic plant tissues and that a transient increase in Lls1 gene expression by about 50 -fold upon physical wounding of maize leaves indicates that the function of Lls1 is regulated in response to stress.

We also found genes encoding miraculin-like protein 2 and miraculin-like protein 3 of Citrus paradise in the CTV infected libraries. In Citrus jambhiri, two distinct transcripts of miraculin-like proteins accumulated to higher levels in leaves after wounding, inoculation with conidia of Alternaria alternata, or treatment with methyl jasmonate vapors (Tsukuda et al., 2006). Stress-inducible genes such as pathogenesis-related class Chitinase (PR3), PR10 (SGR1), Peroxidase prxpr1 (PR9) and a germin-like protein (PR16) were also observed as potentially overexpressed transcripts in CTV inoculated plants.

An important common feature of most PRs is their antifungal effect. Some PR also exhibit antibacterial, insec- 
ticidal, nematicidal and, as recently shown, anti-viral action. PR-10 induced in hot pepper by incompatible interactions with TMV pathotype (TMV-Po) and Xanthomonas campestris was shown to function as a ribonuclease. A hot pepper (Capsicum annuum) cDNA clone encoding pathogenesis-related protein 10 (CaPR-10) was isolated by differential screening of a cDNA library prepared from pepper leaves inoculated with TMV-Po (Park et al., 2004). The inoculation and subsequent phosphorylation of CaPR10 increased its ribonucleolytic activity to cleave invading viral RNAs, and this activity should be important to its antiviral pathway during viral attack in vivo. In the present work, one TC was $S R G 1$, a gene of unknown function that is a member of the PR-10 family (Truesdell and Dickman, 1997) that was also represented in ESTs libraries from cacao leaves treated with inducers of defense response like methyl jasmonate/ethylene (Verica et al., 2004). Xu et al. (2003) showed for the first time that multiple defense responses are specifically induced in Cucumber mosaic virus (CMV) and D satRNA (CMV/D satRNA)-infected tomato plants, but not in mock-inoculated or CMV-infected plants. These responses include callus deposition and hydrogen peroxide accumulation in infected plants. Furthermore, the transcription of several tomato defense-related genes (e.g., PR-1a1, PR-1b1, PR-2, and PR-10) was activated, and the expression of tomato PR-5 and some abiotic and biotic stress-responsive genes are enhanced.

The germin-like protein (PR16) was also observed as overexpressed in CTV inoculated plants. The multifaceted functionality of PR-15 and PR-16, including a cell wall remodeling ability, can be directed against pathogens and may have protective role (Park et al., 2004). Germins and germin-like proteins (GLPs) have been classified as PR-15 and PR-16. PR-16 has been isolated from hot pepper during the resistance response to bacterial and viral infection (Edreva, 2005). Another overexpressed gene in the presence of the CTV was the peroxidase encoding prxr1. Peroxidase prxr1 is considered a PR9 peroxidase that probably strengthens plant cell walls by catalyzing lignin deposition in reaction to microbial attacks (Scherer et al., 2005).

In $P$. trifoliata plants, $P R-2, P R-3, P R-15$ and $P R-16$ gene families were highly expressed within leaves after infection by CTV, whereas no expression was found for other $P R$ gene families (Campos et al., in this issue). According to these authors, the differential $P R$ gene expression profiles vary between infected and healthy tissues, as well as between different pathogen infections. For instance, the high expression of the $P R-3, P R-15$ and $P R-16$ gene families within $P$. trifoliata leaves upon CTV inoculation was found to be suppressed in steam bark after $P$. parasitica infection. This indicates that it is also possible that $P R$ gene expression profiles may vary among tissues.

\section{$B A C$ clones of Ctv}

Citrus tristeza virus (CTV) is an important pathogen of Citrus. A single dominant gene Ctv, present in Poncirus trifoliata, confers broad spectrum resistance against CTV (Gmitter et al., 1996). BAC clones and their use as anchors localized $C t v$ to a 282,699 bp region, comprising 22 predicted genes (Ctv.1 to Ctv.22) (Yang et al., 2003). Refinement of genetic maps delimited this gene to a $121 \mathrm{~kb}$ region, comprising ten candidate $C t v$ resistance genes. The ten candidate genes were individually cloned in an Agrobacterium based binary vector and transformed into three CTV susceptible grapefruit varieties (Rai, 2006). The authors found that two of the candidate R-genes, R-2 and R-3 were exclusively expressed in transgenic plants and in Poncirus trifoliata, while five other genes are also expressed in non-transformed Citrus controls.

In the present work, no significant differences could be observed in the expression profiles of the Ctv regions (Ctv.1 to Ctv.22) of Poncirus trifoliata, challenged or not with CTV. Homologs of Ctv.2, Ctv.3, Ctv.10, Ctv.12, $C t v .15$, Ctv.20, and Ctv.22 were identified in inoculated and non-inoculated Poncirus leaf libraries, as well as in other libraries constructed from Poncirus bark and seeds (data not shown). Moreover, Ctv homologs were also present in libraries constructed from all tissues (leaf, bark, fruit, flower, root, and seed) and all Citrus species analyzed in the CitEST database ( $C$. aurantifolia, $C$. aurantium, $C$. latifolia, $C$. limettioides, $C$. limonia, $C$. reticulata, $\mathrm{C}$. sinensis, and $C$. sunki). It is possible that the $C t v \mathrm{BAC}$ clone regions may be involved in resistance to CTV in $P$. trifoliata, as suggested by Yang et al. (2003) and Rai (2006). Nevertheless, the observation that Ctv homologs seem to be expressed in the related genus Citrus, including in highly susceptible species such as $C$. aurantium, indicates that they are not a major component in resistance, or that they behave in a very unexpected fashion. The Ctv BAC clone regions may not exhibit the characteristics of a typical resistance gene, and it has not been unequivocally shown that they confer resistance to CTV. Hence, further experiments will need to address whether or not the $C t v$ regions play an important role in CTV resistance, and which of them are responsible for the major component of such resistance.

\section{Concluding Remarks}

CTV resistance in P. trifoliata prevents viral proliferation in plants by an undetermined mechanism, essentially resulting in immunity. Lack of a visual hypersensitive response in inoculated plants or in rootstocks with infected susceptible scions suggests that resistance is associated with the interruption of some step in viral multiplication.

Assuming that CTV resistance is monogenic and dominant (Gmitter et al., 1996), we had expected to find evidence of a differentially expressed resistance gene within the CTV-infected library, yet, we could not identify any typical resistance gene. This may be explained by the fact that the libraries were constructed with tissues collected 90 days after infection. In this case, we probably detected only secondary responses to CTV infection. Alternatively, we 
would have to assume an atypical mechanism of resistance that would have to be investigated in further experiments.

\section{References}

Albiach-Marti MR, Grosser JW, Gowda S, Mawassi M, Satyanarayana T, Garnsey S and Dawson WO (2004) Citrus tristeza virus replicates and forms infectious virions in protoplasts of resistant citrus relatives. Mol Breed 14:117-128.

Altschul SF, Madden TL, Schäffer AA, Zhang J, Zhang Z, Miller W and Lipman DJ (1997) Gapped BLAST and PSI-BLAST: A new generation of protein database search programs. Nucleic Acids Res 25:3389-3402.

Audic S and Claverie JM (1997) The significance of digital gene expression profiles. Genome Res 7:986-995.

Chong J, Baltz R, Schmitt C, Beffa R, Fritig B and Saindrenan P (2002) Downregulation of a pathogen-responsive tobacco UDP-Glc:phenylpropanoid glucosyltransferase reduces scopoletin glucoside accumulation, enhances oxidative stress, and weakens virus resistance. Plant Cell 14:10931107.

Edreva A (2005) Pathogenesis-related proteins: Research progress in the last 15 years. Gen Appl Plant Physiology 31:105124.

French CJ, Elder M, Leggett F, Ibrahim RK and Towers GHN (1991). Flavonoids inhibit infectivity of tobacco mosaic virus. Can J Plant Pathol 13:1-6.

Gomez-Gomez L and Carrasco P (1998) Differential expression of the S-adenosyl-L-methionine synthetase genes during pea development. Plant Physiol 117:397-405.

Gmitter FG, Xiao SY, Huang S, Hu XL, Garnsey SM and Deng Z (1996) A localized linkage map of citrus tristeza virus resistance gene region. Theor Appl Genet 92:688-695.

Hajimorad MR and Hill JH (2001) Rsv1-mediated resistance against Soybean mosaic virus- $\mathrm{N}$ is hypersensitive response-independent at inoculation site but has the potential to initiate a hypersensitive response-like mechanism. Mol Plant-Microbe Interact 14:587-598.

Huang X and Madan A (1999) CAP3: A DNA sequence assembly program. Genome Res 9:868-877.

Jaeck E, Dumas B, Geoffroy P, Favet N, Inze D, van Montagu M, Fritig B and Legrand M (1992) Regulation of enzymes involved in lignin biosynthesis: Induction of O-methyltransferase rnRNAs during the hypersensitive reaction of tobacco to tobacco mosaic virus. Mol Plant-Microbe Interact 5:294-300.

Kang BC, Yeam I and Jahn MM (2005) Genetics of plant virus resistance. Annu Rev Phytopathol 43:581-621.

Kawasaki T, Koita H, Nakatsubo T, Hasegawa K, Wakabayashi $\mathrm{K}$, Takahashi H, Umemura K, Umezawa T and Shimamoto K (2006) Cinnamoyl-CoA reductase, a key enzyme in lignin biosynthesis, is an effector of small GTPase Rac in defense signaling in rice. Proc Natl Acad Sci USA 103:230-235.

Kende H (1993) Ethylene biosynthesis. Annu Rev Plant Physiol Plant Mol Biol 44:283-534.

Knoester M, Bol JF, van Loon LC and Linthorst HJ (1995) Virus-induced gene expression for enzymes of ethylene bio- synthesis in hypersensitively reacting tobacco. Mol PlantMicrobe Interact 8:177-180.

Ludwig AA and Tenhaken RA (2001) New cell wall located $\mathrm{N}$-rich protein is strongly induced during the hypersensitive response in Glycine max L. Eur J Plant Pathol 107:323-336.

Mestre PF, Asíns MJ, Carbonell EA and Navarro L (1997) New gene(s) involved in the resistance of Poncirus trifoliata (L.) Raf. to Citrus tristeza virus. Theor Appl Genet 95:691-695.

Park CJ, Kim KJ, Shin R, Park JM, Shin YC and Paek KH (2004) Pathogenesis-related protein 10 isolated from hot pepper functions as a ribonuclease in an antiviral pathway. Plant $\mathbf{J}$ 37:186-98.

Rai M (2006) Refinement of the Citrus tristeza virus resistance gene (Ctv) positional map in Poncirus trifoliata and generation of transgenic grapefruit (Citrus paradise) plant lines with candidate resistance genes in this region. Plant Mol Biol 61:399-414.

Reinhard K and Matern U (1989) The biosynthesis of phytoalexins in Dianthus caryophyllus L. cell cultures: Induction of benzoyl-CoA: Anthranilate N-benzoyltransferases activity. Arch Biochem Biophys 275:295-301.

Scherer NM, Thompson CE, Freitas LF, Bonatto SL and Salzano FM (2005) Patterns of molecular evolution in pathogenesisrelated proteins. Genet Mol Biol 28:645-653.

Truesdell GM and Dickman MB (1997) Isolation of pathogen/stress-inducible cDNAs from alfalfa by mRNA differential display. Plant Mol Biol 33:737-743.

Tsukuda S, Gomi K, Yamamoto H and Akimitsu K (2006) Characterization of cDNAs encoding two distinct miraculin-like proteins and stress-related modulation of the corresponding mRNAs in Citrus jambhiri lush. Plant Mol Biol 60:125-136.

Verica JA, Maximova SN, Strem MD, Carlson JE, Bailey BA and Guiltinan MJ (2004) Isolation of ESTs from cacao (Theobroma cacao L.) leaves treated with inducers of the defense response. Plant Cell Rep 23:404-413.

$\mathrm{Xu}$ P, Blancaflor EB and Roossinck MJ (2003) In spite of induced multiple defense responses, tomato plants infected with $\mathrm{Cu}$ cumber mosaic virus and D satellite RNA succumb to systemic necrosis. Mol Plant-Microbe Interact 16:467-476.

Yang M, Wardzala E, Johal GS and Gray J (2004) The woundinducible $L l s 1$ gene from maize is an orthologue of the Arabidopsis Acdl gene, and the LLS1 protein is present in non-photosynthetic tissues. Plant Mol Biol 54:175-91.

Yang ZN, Ye XR, Molina J, Roose ML and Mirkov TE (2003) Sequence analysis of a 282-kilobase region surrounding the Citrus tristeza virus resistance gene (Ctv) locus in Poncirus trifoliata L. Raf. Plant Physiol 131:482-492.

Ye ZH (1997) Association of caffeoyl CoA 3-O-methyltransferase expression with lignifying tissues in several dicot plants. Plant Physiol 115:1341-1350.

Ye ZH, Zhonga R, Morrison WH 3rd and Himmelsbach DS (2001) Caffeoyl coenzyme A O-methyltransferase and lignin biosynthesis. Phytochemistry 57:1177-1185.

\section{Internet Resources}

MIPS Functional Categories (FunCat), http://mips.gsf.de/projects/ funcat (August 15, 2006).

Associate Editor: Ivan de Godoy Maia 